Original Research Article

\title{
Study on Biological Characteristics of Apricot Blooming in Hongfeng and New Century
}

\author{
Xinting Guan, Yinghui Yuan,Xiujuan Liang \\ College of Plant Science, Luzhou University, Sichuan, China
}

\begin{abstract}
In this paper, the pollination and biology of apricot in Hongfeng and New Century were studied. The results are as follows: (1) The est pollination with the red variety is early, new century's best pollinating varieties is camel yellow. (2) The flowering period of different cultivars was different, and the flowering period of Hongfeng and other varieties was 3 - 7 days later than that of Baxing water apricot and other varieties, which provided germplasm for further breeding of late flowering varieties. (3) Hongfeng, the new century and other varieties of self-flowering rate of $0-3.61 \%$ range, is self-incompatible varieties. (4) The pollen germination rate of different cultivars was higher than 50\%, which indicated that the pollen was mature and the fertility was strong, and the reason of low percentage of self-pollination was pollen abortion, the main reason was self and so on.
\end{abstract}

KEYWORDS: Hongfeng apricot; new century apricot; pollination biology

\section{Introduction}

Apricot produced in China, cultivation has a long history. Spring and Autumn period has been on the almond records, dating at least 2600 years ago, as far away as the ancient peach, $\mathrm{Li}, \mathrm{Li}$ and dates and called 'five fruit', is one of the traditional fruit, has important economic value. The Apricot nutrition and health benefits of high raw food or processing, from domestic and foreign markets, apricot and its processed products is very popular, in short supply. Apricot drought, barren is the mountain greening and ecological agriculture construction of the ideal species, with good ecological benefits. China's apricot germplasm resources are extremely rich, long-term cultivation by the working people practice, has been selected in many places apricot varieties. Such as Jinan red bag apricot, Taian Ba Dan water apricot, Shandong Qingdao Guan Ye face apricot, Beijing camel apricot, Henan Yang Shao apricot, Hebei string stick apricot, Xinjiang color sell apricot, Lanzhou big apricot [1]. These varieties mature early, excellent quality, well received by consumers. However, these varieties of high abortion rate, self-incompatibility (Self-incompatibility, referred to as SI), coupled with the flowering night cream or low temperature often lead to poor pollination and serious impact on the current yield. Li Xiao Sheng in the observation of Lanzhou large apricot flower bud differentiation process found that apricot into the pistil differentiation after showing a variety of pistil dysplasia and distortion form, this apricot deformity and infertility is related. This phenomenon reveals that the analysis of the causes of apricot flower organism provides the exact basis of the occurrence and morphological characteristics of pistil organic distortion. Xie Si-min and other flat on the red robe apricot development of the study shows that the length of the pistil less than $9 \mathrm{~mm}$, are abortion flowers. The degradation rate of pistil in the fruiting branches and middle branches of apricot was low, and the degeneration rate of pistil was high. Flowering temperature is too high despite the flowering ahead, but accelerated the development of flowers and pistil degradation, the formation of more abortion. Apricot in the early flowering season, not entirely more flowers, completely flower pistil is small, and the ovules are small, beads are thin; but in some apricot genotype offspring, late flow often associated with flower bud development. Therefore, the flowering late frost or low temperature, self-incompatibility is the main factor restricting the production of apricot in China, seriously affecting the development of apricot industry [2]. Chen XueSen and other apricot germplasm resources and genetic breeding carried out a more systematic study, and has cultivated HongFeng, the new century and other fine varieties [3]. Further research on the biological characteristics of pollination and pollination of apricot in Hongfeng and New Century, it is necessary to provide technical support for the industrialization of two varieties, so as to produce more economic and social benefits in production. significance.

In general, the study of pollination biology refers to the stage from the beginning of bud differentiation to double fertilization to complete this stage [4]. Therefore, the study of apricot pollination biology mainly includes flower bud 
differentiation, female, stamens abortion, flowering period, and self-incompatibility. A comprehensive understanding of these biological habits and understanding, not only contribute to apricot production, but also for apricot breeding and germplasm innovation to provide scientific basis and reference.

\section{Materials and methods}

Pollination experiment in 2009 in Shandong Province, Tai'an City, Shandong Agricultural University breeding experimental base, the garden for the sandy loam, site conditions and management measures are the same, to eliminate the environmental factors on the varieties and post-representative of the impact of the type of varieties and future generations of the table the difference in type is genetic differences.

\subsection{Tested varieties}

The tested varieties are Hongfeng [5], the new century [6], especially early red, Ba Dan water apricot, two flower troughs, Hongyu apricot, Texas fruit apricot, camel yellow, red purse, Kate, Golden Sun a total of 11 varieties, aged 8 to 10 years, tree vigor, growth results are normal.

\subsection{Pollination test of different varieties}

\subsection{1 - Pollen collection and determination of vitamins}

(1) pollen collection: collecting bell flowering flowers back to the room, with tweezers to remove the anthers, placed in 20 ' $\mathrm{C}$ dry conditions, until the anthers cracked powder, collected in the clean Vial, labeled with the name and collection date, placed in the dryer, placed in the refrigerator $1-2$, save the spare. To solve the problem of inconsistent parents of the flowering, you can cut the male squid, carry into the greenhouse, the end of the branches on the water, remind flowers. Every day at the base of the branches cut a new incision to ensure moisture absorption.

(2) Pollen vitality determination: Peroxidase staining of pollen viability in, pick up a slide, add a small amount of pollen to be tested, 1 drops the-a-naphthol aniline dye, then 1 drops of $0.3 \%$ hydrogen peroxide solution, after 3-5 minutes, covered with a coverslip, use absorbent paper to drain excess water, observed under the microscope. Viability of pollen-containing peroxidase can dye rose red or dark red, without viable pollen yellow or pale yellow, each repeated 3 times, each observation 6 vision.

(3) Pollen germination test: Pollen germination was determined by medium artificial seeding method, media preparation for $15 \%$ sugar, $1 \%$ agar dissolved in distilled water, add a small amount of boric acid, when heated to melt, the medium drops on the slides, take hair DAB a small amount of pollen seeds gently on the culture medium. Seeding will slide into a wet gauze dyeing cylinder, dyeing cylinder 25 constant temperature box culture. Check every 2 hours 1 germination, germination rates are no longer increases so far. 6 vision observed under the microscope, pollen germination of pollen tube elongation exceeds the pollen diameter as standard.

\subsection{2 - Cross-pollination and self-pollination.}

(1) Cross-pollination: emasculation by even the emasculation method, see Chen Xuesen (1989) [7], use the left thumb and forefinger receptacle or the base of the calyx tube, with the right thumb and index finger nails gently sepals, petals and stamens with pinched Off flower torn off stage [8], combined with emasculation, manual method using a small brush dipped in pollen of the delegation on the emasculation of the column, and then bagged, total number of flowers each hybrid pollination, the tone mark, two weeks after the survey percentage.

(2) Self-pollination: For deep cut flowering branch with a flower bud, flower indoors taking powder. Select 1-2 large flowers on each variety, each selected 200-300 flower, with the pollen of the same variety, and then bagged, survey two weeks after flowering fruiting rate [9].

\subsection{3 - Survey items and methods}

(1) Flowering survey: the investigation of the apricot varieties of early flowering, flowering, the end of the flowering and record their time.

(2) Flower quality survey: investigation of Kate, Hong feng, new century, planters, red purse, the second camel yellow, gold Sun, Ba Danshui apricots, early red and other varieties of the same Treaty 300-400/flower varieties listed statistics total number of flowers and utterly spent. Flower grading standards: pistil high above the Stamen or pistils and stamens, to fully flower pistil below the Stamen or pistils degenerate into sterile flowers. 
(3) Fruit rate survey: to investigate the pollination combination of fruit set, including the natural fruit set rate and artificial pollination fruit set fruit rate.

\section{Results and Analysis}

\subsection{Comparison of flowering period, effective flower and natural fruit setting rate}

Almond trees flowering early, vulnerable to late frost damage. The late flowering varieties are one of the targets of apricot breeding; and the effective ratio of flowers and natural fruit rate is an important factor in determining whether a variety is high yield. For the new century, Hongfeng, Ba Dan water apricot and other 11 varieties of flowering, effective flower ratio and natural fruit rate of the survey results in Table 1.

It can be seen from Table 1: different apricot varieties flowering period has some differences in Hongfeng, New Century and other varieties of the flowering of the latest; but the difference between the larger, according to the survey, in 1999, Hongfeng flowering $(\mathrm{P}<0.05)$. However, in 2003, the two flowering periods were only two days difference. The ratio of the effective flower in the survey was the highest (45\%), Hong Feng $(40 \%)$,

\begin{tabular}{|l|l|c|c|c|}
\hline \multicolumn{1}{|c|}{ Variety } & $\begin{array}{c}\text { Early flowering } \\
\text { (day / month) }\end{array}$ & $\begin{array}{c}\text { Last flowering } \\
\text { (day / month) }\end{array}$ & $\begin{array}{c}\text { Effective flower } \\
\text { ratio (\%) }\end{array}$ & $\begin{array}{c}\text { Natural fruit } \\
\text { setting (\%) }\end{array}$ \\
\hline Barton water apricot & $23 / 3$ & $30 / 3$ & 23 & 1.6 \\
\hline Hongyu Xing & $24 / 3$ & $31 / 3$ & 33 & 18.4 \\
\hline Camel yellow & $24 / 3$ & $30 / 3$ & 10 & 1.2 \\
\hline Two flower beds & $26 / 3$ & $2 / 4$ & 33 & 11.1 \\
\hline Texas fruit apricot & $29 / 3$ & $4 / 4$ & 35 & 16.0 \\
\hline Kate & $25 / 3$ & $1 / 4$ & 45 & 31.5 \\
\hline Golden Sun & $24 / 3$ & $29 / 3$ & 38 & 28.5 \\
\hline Red purse & $23 / 3$ & $30 / 3$ & 9 & 2.1 \\
\hline Hongfeng & $31 / 3$ & $6 / 4$ & 40 & 24.1 \\
\hline New Century & $30 / 3$ & $6 / 4$ & 29 & 21.3 \\
\hline Extra Early Red & $24 / 3$ & $31 / 3$ & 38 & 16.1 \\
\hline
\end{tabular}

Table 1. Some apricot flower, flower ratio and natural fruit setting rate

Red purse $(9 \%)$, and effective spending rate was positively correlated with natural fruit set $(r=0.92)$. Effective to spend the higher the ratio, higher fruit set and yield strength. Genetic control of flower on the one hand, and breed, and was greatly influenced by tree nutrition, production, enhance fertilizer and pruning, raise the level of tree nutrition, is to reduce the ratio of sterile flowers, major measures to ensure high yield and quality of apricot [10].

\subsection{Comparison of pollen viability and germination}

\begin{tabular}{|l|c|c|}
\hline Variety & Pollen vitality (\%) & Pollen germination (\%) \\
\hline Hongfeng & 83 & 79 \\
\hline New century & 79 & 65 \\
\hline Extra early red & 83 & 60 \\
\hline Two flower vase & 84 & 57 \\
\hline Red Purse & 84 & 51 \\
\hline Ba Dan water apricot & 86 & 52 \\
\hline Hongyu Xing & 83 & 58 \\
\hline Texas fruit apricot & 87 & 74 \\
\hline Camel Yellow & 77 & 61 \\
\hline Golden Sun & 81 & 55 \\
\hline Kate & 80 & 59 \\
\hline
\end{tabular}

Table 2. Comparison of pollen viability and germination of some apricot cultivars 
It can be seen from Table 2 that the pollen viability of the tested cultivars is high, most of them are higher than $80 \%$, and the difference is not big between them. The pollen vitality of apricot is the highest. But the germination rate of pollen was different, and the pollen germination rate of Hongfeng was the highest, while the pollen germination rate of red lotus was the lowest, and the pollen germination rate of the tested varieties was higher than $50 \%$, which indicated that pollen was mature and Strong, excluding some apricot self-flowering low rate of reason is pollen abortion, the main reason may be caused by self-incompatibility [11].

\begin{tabular}{|l|c|c|c|c|}
\hline Variety & Treatment & Number of fruits & Set fruit rate (\%) & Self-esteem type \\
\hline Hongfeng & 181 & 0 & 0.00 & SI \\
\hline New century & 138 & 5 & 3.61 & SI \\
\hline Kate & 31 & 11 & 35.5 & SC \\
\hline Extra early red & 333 & 12 & 3.61 & SI \\
\hline Ba Dan water apricot & 214 & 0 & 0.00 & SI \\
\hline Red Purse & 276 & 0 & 0.00 & SI \\
\hline Two flower troughs & 152 & 1 & 0.66 & SI \\
\hline HongyuXing & 324 & 2 & 0.62 & SI \\
\hline Texas fruit apricot & 269 & 2 & 0.74 & SI \\
\hline Camel Yellow & 175 & 0 & 0.00 & SC \\
\hline Golden Sun & 146 & 14 & 13.7 & \\
\hline
\end{tabular}

Note: Self-pollination rate $\geqslant 6 \%$ for self-compatibility (SC). $<6 \%$ for self-incompatibility (SI)

Table 3. Hongfeng, the new century and other 11 varieties of self-pollination test results

It can be seen from Table 3, self-pollination fruit set rate is generally low, which Kate and Golden Sun's selfpollination fruit set higher rate, are self-compatible varieties, other varieties of fruit set rate of less than $6 \%$ Unmatched breed. In view of this, the breeding of good quality, self-cultivated meat and new varieties of apricot, is China's apricot breeding research in one of the important issues.

\subsection{Effects of different varieties of pollination on fruit setting in Hongfeng and New Century}

\begin{tabular}{|c|c|c|c|c|}
\hline Variety & Pollination tree species & $\begin{array}{l}\text { Handle the number } \\
\text { of flowers }\end{array}$ & $\begin{array}{l}\text { Fruit } \\
\text { number }\end{array}$ & Fruit set rate (\%) \\
\hline & Hong Feng & 181 & 1 & 0.55 \\
\hline & New Century & 289 & o & 0.00 \\
\hline & Extra early red & 192 & 90 & 46.87 \\
\hline & Barton water apricot & 202 & 43 & 21.48 \\
\hline & Two flower pots & 350 & 150 & 42.85 \\
\hline Hong & CamelYellow & 194 & 38 & 19.85 \\
\hline \multirow[t]{9}{*}{ Feng } & Hongyu Xing & 208 & 76 & 36.54 \\
\hline & Texas fruit apricot & 128 & 37 & 28.91 \\
\hline & Red purse & 241 & 4 & 1.66 \\
\hline & Kate & 222 & 69 & 31.08 \\
\hline & Golden Sun & 252 & 47 & 18.65 \\
\hline & Hong Feng & 275 & 2 & 0.73 \\
\hline & New Century & 138 & o & 0.00 \\
\hline & Extraearly red & $\mathbf{3 3 3}$ & 12 & 3.61 \\
\hline & Ba Dan water apricot & 246 & 32 & 13.01 \\
\hline New & Two flower pots & 230 & 21 & 9.13 \\
\hline \multirow[t]{6}{*}{ Century } & CamelYellow & 104 & 33 & 31.73 \\
\hline & Hongyu Xing & 131 & 9 & 6.87 \\
\hline & Texas fruit apricot & 146 & 14 & 9.59 \\
\hline & Red purse & 274 & 2 & 0.73 \\
\hline & Kate & 214 & 17 & 7.94 \\
\hline & Golden Sun & 352 & 69 & 19.61 \\
\hline
\end{tabular}

Table 4. Different varieties of pollination on Hongfeng, the new century fruit rate

As can be seen from Table 4, Hongfeng, the new century, the pollination rate of each other is $0-0.73 \%$, mainly because they are selected from the common parents of the sister line, S genotype similar or the same [12]. Therefore, to Hongfeng, the new century-based varieties of apricot, to configure other varieties as pollination tree. (2) Pollination of different apricot cultivars were pollinated to Hongfeng and New Century respectively. Which Hongfeng to early red for 
the pollination tree fruit set the highest rate of 46.87 , followed by two flower trough, fruit set rate of 42.85 , therefore, particularly early red and two flower trough is the ideal pollination tree Hongfeng. In the new century, camel yellow was the highest fruit rate of 31.73, followed by Golden Sun, 19.61, and it was found that camel yellow and golden sun were better in the new century.

\section{Discussion}

\subsection{Hongfeng, the best pollination tree species in the new century}

The effective pollination period (EPP) of fruit trees is limited by many factors, including the ability to accept stigma, physiological status of pistil and embryo maturity. These constraints depend on genetic, environmental or cultivation factors (Burgos Letal, 1991). Under the warm climate conditions of the southern Mediterranean, the effective pollination period of the apricot is relatively short, and the normal duration is 4 days, if the pollination is on the 6th day, the fruit setting rate will drop sharply. Stigma sensation is similar to EPP. In general, the limiting factor for EPP is short stigma sensibility, whereas other factors will limit EPP when climate conditions and breed's behavior maintain good stigma sensation after 6 days. The study found that apricot (cv.Moniquifino) 8 days after the flowering ovule mature, in the nonpollinated flowers in the development of ovules more slowly, EPP is mainly limited by the rapid development of embryo sac. Burgos L (1995) have shown that the lack of susceptibility to pollen germination and pollen elongation after pollination of female flowers can also affect fruit setting. In addition, optimum pollination time is not always associated with the flowers open, flower temperature is too high, accelerated the degradation of flower development and the pistil, severely limiting effective pollination period (Burgos L,1991). Zhao Xiping (1994) study apricot flower pistil stigma receptive to open half an hour at its best, best of times flowers after pollination in 4 hours, and pollination has some strong within a 48-hour rate; Burgros 1 (1991) argues that the apricot flower stamens ripe, both after the flowers stigmas mature. After flowering 2D 1d percentage higher than the flowers after pollination, degradation of hot temperature accelerates the pistil, shortens the effective pollination time. This difference may be due to differences in species and climate.

Lv Zengren and others (1992) found that the lack of suitable pollination varieties is one of the important reasons for the low seed setting rate. An excellent pollination variety, in addition to its main varieties with good hybrid compatibility, but also should have good pollen vitality. Pollen viability and germination rate were positively correlated. The germination rate of pollen at high temperature was faster than that at low temperature. The optimum germination temperature of pollen was 20 - 25. Zhang Jianguang (1990) found that apricot pollen $2 \mathrm{~h}$ after pollination can germinate into the stigma, 50h into the ovary, $4 \mathrm{~d}$ after pollination fertilization. After fertilization soon zygote is split, $4 \mathrm{~d}$ formation of small embryo. Apricot pistil takes pollen for a long time, but pollen germination rate is high after flowering.

The results showed that the best pollinated varieties of Hongfeng were the early red and the best pollinated varieties in the new century were camel yellow.

\subsection{Hongfeng, the flowering characteristics of the new century}

Deciduous fruit flowering period, mainly by the control of two factors, one is cooling capacity, the second is the accumulation of heat in spring. The flowering is generally predicted by calculating the Chilling Units (CU) required for boring and the Growing Degree Hours (GDH) required for flowering. There is a negative correlation between these two values, that is, more heat can be partially compensated for the amount of heat required (Garcia Getal, 1999). Plants that are dormant or partially subjected to cold-temperature treatments require more calorie units to blossom than plants that have met the cold-warm requirement. On the contrary, when the completion of dormancy and get more cold, you can reduce the amount of heat required for flowering units. It is observed that there is a big difference in the early spring of different years, the biggest difference is more than a month. The length of flowering period was negatively correlated with early flowering and temperature, and high temperature promoted flowering. According to the flowering period, the observed cultivars can be divided into three types, early flower varieties, middle flower varieties and late flowering varieties (Szalay Letal, 2000). Breeding of late spring varieties can reduce the chance of early spring frost damage, the production of almond trees is of great significance (Chen Xuesen, 1996), has been selected Hongfeng and the new century and other early maturing late spring varieties (Chen Xuesen, 2001).

Flower organ abortion is a common phenomenon in the process of flower bud formation in fruit trees, including various stages of development of pistil (such as elongated, curved, withered short or missing) and staminal abortion (mainly anther degeneration, no pollen and pollen abortion). Chinese apricot varieties of pistil abortion is particularly serious. Li Yousheng (1986) observed in the process of differentiation of apricot flower buds in Lanzhou, apricot showed a variety of pistil dysplasia and distortion form, this apricot deformity and infertility is related. This phenomenon reveals that the analysis of the causes of apricot flower organism provides the exact basis of the occurrence and morphological characteristics of pistil organic distortion. Xie Si-min et al, (1994) showed that the length of the pistil was less than $9 \mathrm{~mm}$, are aborted flowers. The degradation rate of pistil in the fruiting branches and middle branches of apricot was 
low, and the degeneration rate of pistil was high, the difference is remarkable. Flowering temperature is too high despite the flowering ahead, but accelerated the development of flowers and pistil degradation, the formation of more abortion. Apricot in the early flowering season, not entirely more flowers, full of pistil is relatively small, and the ovules are small, beads are thin; but in Hungary and Romania apricot genotype offspring, late flowers often associated with flower bud development (Bassi Detal, 1995). The results of the survey show that the female flower abortion on the one hand by genetic constraints, and tree nutrition (Chen Xuesen, 2000). Apricot species and varieties, nutrient levels and changes in the process of flower bud differentiation are the main external factors that affect the formation of incomplete flowers. The nutritional status of the pros and cons affect the degree of incomplete flowers, nutritional status is good, less abortion, and vice versa (Lu Zengren, 1992). Therefore, through breeding and reasonable agricultural technical measures (shaping pruning, soil and fertilizer management, application of growth regulators, etc.) can reduce the apricot flower, improve fruit set rate.

In Tai'an apricot producing areas, apricot blossom earlier, vulnerable to late frost damage. Flowering night cream is often a direct result of flower organ damage, resulting in reduced production. Therefore, the appropriate delay in flowering production is extremely beneficial to almond trees.

The results showed that the flowering period of different cultivars was different, and the flowering period of Hongfeng and New Century was 3 - 7 days later than that of Ba Dan water apricot and other varieties, which provided germplasm for further breeding of late flowering varieties.

\subsection{Hongfeng, self-incompatibility in the new century}

In recent years, the results show that in more than 2,000 apricot varieties of resources, self-compatibility and selfincompatible varieties exist at the same time. North China ecological varieties of species, mostly self-incompatible, but there are individual varieties of species can be self-sturdy. Most varieties of European ecological species (including Europe, North America, South Africa and Australia) are self-friendly, but in recent years some breeds have been found to be self-incompatible (Burgos Letal, 1997). Self-pollinated pollen tube growth slower than when crossed pollination, most pollen tubes stopped growing halfway, and only a few reached the ovules. Apricot cultivar self-pollination test of Tokalogolu showed that the pollen tube could germinate well, enter and penetrate the style, but the pollen tube could not penetrate the ovule and could not finish the fertilization. The results showed that some pollinated combinations of apricot varieties showed positive and negative crosses, some of them were affinity, others were not compatible, and there were significant differences in the combination of positive and negative crosses (Lv Zengren et al., 1996; Wang Yuzhu et al., 1998). Zhao et al. (1995) studied the pollination characteristics of the apricot and the parent and the offspring, and found that the offspring had a better affinity for the female parent with a certain self-filling ability. Soybean lines were pollinated each other showed a low seed setting rate, which shows that the relationship between pollinating affinity and genetic relationship.

The results showed that the self-flowering ability of Hongfeng and New century was in the range of $0-3.61 \%$, and the pollen germination rate was higher than $50 \%$, which was that the pollen abortion was mainly due to the low seed setting rate Self-incompatibility.

\section{References}

1. Li Xianli, Zhang Yanmin, et al. Advances in Research on Apricot Germplasm Resources and Genetic Breeding [J]. Journal of Fruit Science, 2001,18 (3): 178 - 181

2. Chen Xuesen. Advances in Biotechnology Research on Fruit Trees [J]. Journal of Shandong Agricultural University, 1994, 25 (Suppl): 177 - 182

3. Gao Dongsheng, Li Xianli, et al. Breeding of a new early line of apricot [J]. Shanxi Fruit Tree, 1999, 4: 5 - 6

4. Chen Xuesen, Liang Huairui. Advances in pollination and biology of fruit trees [J]. Journal of Shandong Agricultural University, 2000, 31 (3): 345 - 348

5. Li Xianli, et al. New varieties of early apricot apricot cultivars - Hongfeng [J]. Journal of Horticulture, 2001,28 (6): 575 - 576

6. Chinese Journal of Horticulture, 2001,28 (5): 475 - 476

7. Chen Xuesen et al. Plant Breeding Experiment [M]. Beijing, Higher Education Press, 2004: 146 - 148

8. Li Zheng implicit, Huang Chai, et al. Apricot flower bud differentiation observation [J]. Horticulture, 2008,5 (1): 68 - 70

9. Journal of Horticulture, 1992,19 (1): 7 - 10 (in Chinese with English abstract) [J].

10. Journal of Shanxi Agricultural University, 1994, 14 (1): 60 - 63 [J]. Journal of Shanxi Agricultural University, 1994,14 (1): 60 $-63$

11. Burgos L, Ledbetter C.A. Egea, J, et al .Inheritance of sexual incompatibility in apricot [J]. Plant Breeding 1997, 166: 383 386

12. Egea J, Burgos L. Fertile pollination period as related to stigma receptivity in apricot [J]. Scientia Horticulturae, $1992,52: 77$ 83 